\title{
Taurine supplementation reduces oxidative stress and protects the liver in an iron-overload murine model
}

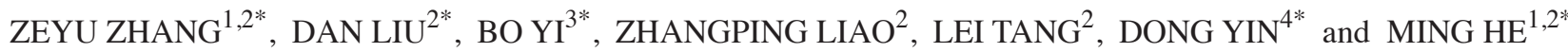 \\ ${ }^{1}$ State Key Laboratory of Food Science and Technology, Nanchang University, Nanchang, Jiangxi 330047; \\ ${ }^{2}$ Department of Pharmacology and Molecular Therapeutics, Nanchang University School of Pharmaceutical Science, \\ Nanchang, Jiangxi 330006; ${ }^{3}$ Second Abdominal Surgery Department, Jiangxi Province Tumor Hospital, Nanchang, \\ Jiangxi 330029; ${ }^{4}$ Jiangxi Provincial Key Laboratory of Molecular Medicine at the Second Affiliated Hospital, \\ Nanchang University, Nanchang, Jiangxi 330006, P.R. China
}

Received August 22, 2013; Accepted May 14, 2014

DOI: $10.3892 / \mathrm{mmr} .2014 .2544$

\begin{abstract}
We previously demonstrated that iron overload induces liver damage by causing the formation of reactive oxygen species (ROS). Taurine is a potent free radical scavenger that attenuates the damage caused by excessive oxygen free radicals. Therefore, the aim of the present study was to investigate whether taurine could reduce the hepatotoxicity of iron overload with regard to ROS production. Mice were intraperitoneally injected with iron 5 days/week for 13 weeks to achieve iron overload. It was found that iron overload resulted in liver dysfunction, increased apoptosis and elevated oxidative stress. Taurine supplementation increased liver taurine levels by $40 \%$ and led to improved liver function, as well as a reduction in apoptosis, ROS formation and mitochondrial swelling and an attenuation in the loss of the mitochondrial membrane potential. Treatment with taurine mediated a reduction in oxidative stress in iron-overloaded mice, attenuated liver lipid peroxidation, elevated antioxidant enzyme activities and maintained reduced glutathione levels. These results indicate that taurine reduces iron-induced hepatic oxidative stress, preserves liver function and inhibits hepatocyte apoptosis. Therefore, taurine may be a potential therapeutic drug to reduce liver damage caused by iron overload.
\end{abstract}

\section{Introduction}

Secondary iron overload and primary hemochromatosis have received increasing attention due to worldwide epidemics (1-3).

Correspondence to: Professor Ming He, Department of Pharmacology and Molecular Therapeutics, Nanchang University School of Pharmaceutical Science, 461 Bayi Road, Nanchang, Jiangxi 330006, P.R. China

E-mail: jxhm56@hotmail.com

${ }^{*}$ Contributed equally

Key words: taurine, iron overload, liver, oxidative stress, apoptosis
Iron is deposited in numerous tissues, most notably the liver, where it can lead to significant organ damage (4). Iron overload in the liver is observed in patients with chronic liver diseases, including alcoholic liver disease and chronic viral hepatitis, and is a secondary side effect of repeated blood transfusions (5-7). Emerging evidence suggests that oxidative stress, mediated by free radicals and reactive oxygen species (ROS), may have a role in the pathophysiology of iron-induced liver injury and cell death $(8,9)$. In a previous study we found that iron overload initiates excessive ROS formation, and that the liver is severely damaged as a result of this oxidative stress (10). However, little is known about the use of antioxidants as possible preventive or curative agents in excessive iron-induced liver damage.

Taurine, a sulfur-containing amino acid, is present at high concentrations in the liver (11). Numerous studies have found that taurine has a protective effect against chemically-induced hepatotoxicity (12-15). Furthermore, taurine has been reported to act as an antioxidant in biological systems. As an antioxidant, taurine has the ability to scavenge ROS and attenuate lipid peroxidation and, as a consequence, stabilizes biological membranes $(16,17)$.

Since taurine has been shown to have potent antioxidant properties, its role in hepatotoxicity induced by iron overload was investigated in the present study. The status of the physiological parameters associated with hepatotoxicity induced by iron overload, including the levels of intracellular antioxidant enzymes, the glutathione (GSH)/glutathione disulfide (GSSG) ratio and the involvement of the mitochondria-dependent apoptotic pathway, was assessed, either with or without supplementation of taurine. The results of this may provide critical information for the treatment of oxidative stress mediated by iron overload in the liver.

\section{Materials and methods}

Experimental animals and iron overloading protocols.

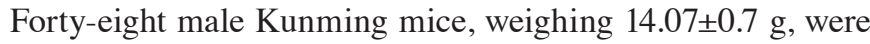
obtained from Nanchang University (Nanchang, China). The mice were randomly divided to receive either taurine $(0.1 \mathrm{~mol} / \mathrm{l})$ or vehicle $(2.5 \%$ dextrose $)$ in their drinking water 2 weeks prior to the initial injections and throughout 
the course of the experiments. The mice were continuously injected with either iron or placebo ( $0.1 \mathrm{ml} 10 \%$ dextrose) for a total of 13 weeks (total dose, $200 \mathrm{mg} / 25 \mathrm{~g}$ body weight) as previously described (18). Mice were thus divided into the placebo plus vehicle, placebo plus taurine, iron plus vehicle and iron plus taurine groups. All care and experiments in this study conformed to the National Institutes of Health (NIH) Guide for Care and Use of Laboratory Animals (NIH publication 86-23, revised in 1986). The use of animals was reviewed and approved by the Nanchang University Animal Care Review Committee. At the end of the experiment the mice were sacrificed by cervical dislocation and blood was collected by cardiac puncture. The liver was immediately excised, weighed and divided for further analysis.

Determination of iron levels in the serum and liver. The iron concentration in the serum was determined using an assay based on the generation of an iron-ferrozine complex, as described by Galleano and Puntarulo (19). The iron concentration in the digested liver sample was measured using a spectrophotometer $(535 \mathrm{~nm})$ following the addition of $2 \mathrm{mM}$ bathophenanthroline disulfonic acid.

Determination of aspartate transaminase (AST) and alanine transaminase (ALT) levels. Serum levels of AST and ALT were measured using an autoanalyzer (Roche Cobas Integra 400, Roche Diagnostics, Holliston, MA, USA) and AST and ALT reagent kits from Roche Diagnostics (Indianapolis, IN, USA).

Determination of taurine levels. The liver tissue was homogenized and the supernatant was ultra-filtered and diluted with methionine sulfone (an internal standard). The taurine concentration was determined as previously described using a high-performance liquid chromatography system and a specific Pico-Tag column (Waters Corp., Milford, MA, USA) (20).

Terminal deoxynucleotidyl transferase-mediated dUTP nick end labeling (TUNEL) assay. The TUNEL assay was performed using a commercially available kit (Promega Corp., Madison, WI, USA) in accordance with the manufacturer's instructions. Briefly, liver samples were fixed by perfusion with $10 \%$ buffered formalin, and 5- $\mu \mathrm{m}$ paraffin-embedded sections were obtained. The sections were then subjected to a DeadEnd ${ }^{\mathrm{TM}}$ Colorimetric TUNELSystem assay. The paraffin-embedded liver sections were re-treated to remove paraffin and fixed again in $4 \%$ paraformaldehyde. Following permeabilization, the sections were labeled with a terminal transferase reaction mix and bound to streptavidin horseradish peroxidase (HRP). The sections were then incubated with chromogen HRP and subsequently treated with diaminobenzidine and counterstained lightly with hematoxylin. Brown nuclei with nuclear condensation in the stained cells were considered to be TUNEL positive. Hepatocyte apoptosis in the liver sections was quantified by counting the number of TUNEL-positive cells in a random microscopic low-power field (magnification, x400).

Determination of lipid peroxidation and antioxidant enzyme activities.

Malondialdehyde (MDA). The lipid peroxide content in the liver was determined by quantifying the thiobarbituric acid reactive substances as previously described (21). Briefly, $0.5 \mathrm{ml}$ supernatant was mixed with $1.5 \mathrm{ml}$ thiobarbituric acid, $1.5 \mathrm{ml}$ acetic acid ( $\mathrm{pH} 3.5), 0.2 \mathrm{ml}$ sodium dodecyl sulfate and $0.5 \mathrm{ml}$ distilled water, and the samples and standards were heated to $100^{\circ} \mathrm{C}$ for $1 \mathrm{~h}$. The absorbance was measured at $532 \mathrm{~nm}$ on a spectrophotometer. Commercially available MDA was used as a standard.

Superoxide dismutase (SOD). SOD activity in the tissue homogenate was measured following the method previously described by Beauchamp and Friedrich (22), with slight modifications. The reaction mixture $(0.8 \mathrm{ml})$, containing $100 \mu \mathrm{mol} / 1$ xanthine, $100 \mu \mathrm{mol} / 1$ EDTA, $25 \mu \mathrm{mol} / 1$ nitroblue tetrazolium (NBT) and $50 \mathrm{mmol} / 1 \mathrm{Na}_{2} \mathrm{CO}_{3}(\mathrm{pH} \mathrm{10.2)}$, was added to $0.1 \mathrm{ml}$ liver homogenate. After $10 \mathrm{~min}$ preincubation at room temperature, the reaction was initiated by the addition of $0.1 \mathrm{ml}$ xanthine oxidase $(0.05 \mathrm{U} / \mathrm{ml})$, and the absorbance at $560 \mathrm{~nm}$ was recorded every $30 \mathrm{sec}$ for $5 \mathrm{~min}$. A standard curve for SOD activity was assayed spectrophotometrically as the inhibition of the photochemical reduction of NBT at $560 \mathrm{~nm}$.

GSH-peroxidase (GSH-Px). GSH-Px activity was determined as previously described, with slight modifications (23). The liver tissue was homogenized (1:10) in $75 \mathrm{mmol} / \mathrm{l}$ phosphate buffer ( $\mathrm{pH}$ 7.0). The homogenate was then centrifuged at $20,000 \mathrm{x} \mathrm{g}$ for $25 \mathrm{~min}$, and the supernatant was aspirated and assayed for total cytosolic GSH-Px activity. GSH-Px activity was assayed in a $3-\mathrm{ml}$ cuvette containing $2.0 \mathrm{ml} 75 \mathrm{mmol} / 1$ phosphate buffer ( $\mathrm{pH}$ 7.0). The following solutions were then added: $50 \mu \mathrm{l}$ glutathione $(60 \mathrm{mmol} / \mathrm{l}), 100 \mu \mathrm{l}$ glutathione reductase solution $(30 \mathrm{U} / \mathrm{ml}), 50 \mu 1 \mathrm{NaN}_{3}(0.12 \mathrm{~mol} / \mathrm{l}), 100 \mu \mathrm{l}$ $\mathrm{Na}_{2}$ EDTA (15 mmol/l), $100 \mu \mathrm{l}$ reduced nicotinamide adenine dinucleotide phosphate (NADPH; $3.0 \mathrm{mmol} / \mathrm{l}$ ) and $100 \mu \mathrm{l}$ cytosolic fraction. The reaction was initiated by the addition of $100 \mu \mathrm{l} 7.5 \mathrm{mmol} / \mathrm{l} \mathrm{H}_{2} \mathrm{O}_{2}$, and the conversion of NADPH to NADP was monitored by recording the change in the absorbance at $340 \mathrm{~nm}$ at 1-min intervals for $5 \mathrm{~min}$. The GSH-Px activity was expressed as the quantity of reduced NADPH (in nanomoles) oxidized to NADP per minute per milligram of protein, with a molar extinction coefficient for NADPH at $340 \mathrm{~nm}$ of $6.22 \times 10^{6}$.

Catalase. Catalase activity was determined as previously described (23). The liver tissue was homogenized (1:10) in $50 \mathrm{mmol} / \mathrm{l}$ potassium phosphate buffer ( $\mathrm{pH} 7.4$ ), and the homogenate was centrifuged at $40,000 \mathrm{x} g$ for $30 \mathrm{~min}$. Approximately $50 \mu \mathrm{l}$ supernatant was added to a 3 ml-cuvette containing $2.95 \mathrm{ml}$ hydrogen peroxide $(19 \mathrm{mmol} / \mathrm{l})$ in $50 \mathrm{mmol} / 1$ potassium phosphate buffer ( $\mathrm{pH} 7.4)$. Changes in absorbance at $240 \mathrm{~nm}$ were recorded continuously for $5 \mathrm{~min}$. Catalase activity was expressed as units per milligram of protein.

Measurement of total glutathione (GSSG+GSH) levels (reduced and oxidized). The concentration of GSSG+GSH in the liver was measured using the glutathione reductase/5,5'-dithiobis-(2-nitrobenzoic acid) (DTNB) recycling assay (24). The rate of 5'-thio-2-nitrobenzoic acid formation was measured at an absorbance of $412 \mathrm{~nm}$ and was proportional to the sum of GSH+GSSG present. Liver tissue was homogenized in 5\% sulfosalicylic acid, and the homogenate was centrifuged for $10 \mathrm{~min}$ at $10,000 \mathrm{x} \mathrm{g}$. The supernatant was stored at $4^{\circ} \mathrm{C}$ until assayed. GSSG alone was measured by treating the sulfosalicylic acid supernatant with 
2-vinylpyridine and triethanolamine. The solution was vigorously mixed, and the final $\mathrm{pH}$ of the solution was adjusted to between six and seven. After $60 \mathrm{~min}$, the samples were assayed as described above in the DTNB-GSSG reductase recycling assay. The GSH values were calculated as the difference between the total (GSSG+GSH) and GSSG concentrations. The values are reported in GSH equivalents and expressed as micromoles per gram of tissue.

Hepatocyte preparation. Hepatocytes were isolated using a two-step collagenase perfusion method. Following mechanical disruption of the liver capsule, liver cells were collected in Williams' Medium E and serially filtered (30-,50- and 80-mesh) through an 85-ml Cellector (Bellco Biotechnology, Vineland, NJ, USA) tissue sieve. Between $10 \times 10^{6}$ and $25 \times 10^{6}$ cells were obtained from a single mouse liver.

Measurement of intracellular ROS. The fluorescent probe 2',7'-dichloro-dihydro-fluorescein diacetate (DCFH-DA) is converted by intracellular esterases to DCFH, which is then oxidized by ROS to highly fluorescent DCF. The ROS Detection Reagent assay (Invitrogen Life Technologies, Carslbad, CA, USA) was performed in accordance with the manufacturer's instructions. Hepatocytes were washed twice with cold phosphate-buffered saline (PBS) and incubated in Dulbecco's Modified Eagle's Medium containing $10 \mu \mathrm{M}$ DCFH-DA (Invitrogen Life Technologies). Following centrifugation at $800 \mathrm{x} \mathrm{g}$ for $5 \mathrm{~min}$ and two washes with cold PBS, the fluorescence intensity of each group was determined using flow cytometric analysis (Becton-Dickinson, Franklin Lakes, NJ, USA) at excitation and emission (ex/em) wavelengths of $485 / 528 \mathrm{~nm}$, respectively.

Assessment of the mitochondrial membrane potential $(\Delta \psi)$. The mitochondrial membrane potential was assessed using the fluorescent dye JC-1 (Invitrogen Life Technologies), a lipophilic cationic dye that selectively enters mitochondria and reversibly changes color from green to red when the $\Delta \psi$ increases (25). Therefore, the ratio of the red to green fluorescent intensity of the cells reflects the $\Delta \psi$. Suspended hepatocytes were incubated with JC-1 (200 $\mu \mathrm{l})$ for $20 \mathrm{~min}$ at $37^{\circ} \mathrm{C}$ followed by two washes with PBS to remove the excess dye. Fluorescence was then measured using a flow cytometer (Becton-Dickinson) with ex/em wavelengths of 530/580 nm (red), and 485/530 nm (green).

Preparation of mitochondrial fraction. Mitochondria were isolated using conventional differential centrifugation from the liver of mice that had fasted overnight. The livers were homogenized in $250 \mathrm{mM}$ sucrose, $1 \mathrm{mM}$ ethylene glycol tetraacetic acid (EGTA) and 10 mM HEPES buffer (pH 7.2). The mitochondrial suspension was washed twice in the same medium containing $0.1 \mathrm{mM}$ EGTA, and the final pellet was resuspended in $250 \mathrm{mM}$ sucrose. The final protein concentration was $80-100 \mathrm{mg} / \mathrm{ml}$, as measured by the Biuret method, using bovine serum albumin as the protein standard.

Mitochondrial swelling. The swelling experiments were performed as previously described by Beavis et al (26) using a standard medium containing $125 \mathrm{mM}$ sucrose, $10 \mathrm{mM}$
HEPES buffer ( $\mathrm{pH} 7.2), 2.5 \mathrm{mM}$ succinate and $4.0 \mathrm{mM}$ rotenone at $25^{\circ} \mathrm{C}$. The final volume used was $1.0 \mathrm{ml}$, and the protein concentration was $\sim 0.5 \mathrm{mg} / \mathrm{ml}$. Changes in absorbance at $520 \mathrm{~nm}$ were monitored in a thermostatically controlled Hitachi U 2000 spectrophotometer (Hitachi, Ltd., Tokyo, Japan).

Statistical analysis. Data analysis was performed using SPSS ${ }^{\circledR}$ statistical software version 11.0 (SPSS Inc., Chicago, IL, USA). The data are expressed as the mean \pm standard error of the mean from $\geq 12$ independent experiments. Each treatment was performed in triplicate culture wells. The differences between the means of each group were tested using a one-way analysis of variance followed by the Student-Newman-Keuls test to compare between multiple groups. $\mathrm{P}<0.05$ was considered to indicate a statistically significant difference.

\section{Results}

Taurine improves the liver-to-body ratio, as well as the ALT and AST levels, without affecting iron accumulation. Serum and hepatic iron levels were significantly increased in all iron-overloaded mice, regardless of taurine supplementation. To investigate whether liver injury and dysfunction were caused by iron overload, the liver-to-body weight ratio (\%) and the levels of serum ALT and AST, which are important markers of dysfunction, were analyzed. Iron-overloaded mice showed a 1.9-fold increase in the liver-to-body weight ratio, and a 4.5- and 3.7-fold elevation in the serum ALT and AST levels, respectively. However, treatment with taurine was found to suppress these changes (Table I).

Taurine prevents apoptosis in iron-overloaded mice. In mice that did not receive iron treatment, only a few TUNEL-positive hepatocytes were identified; however, numerous TUNEL-positive hepatocytes were observed in the iron-overloaded animals. Taurine supplementation significantly reduced the total number of TUNEL-positive hepatocytes to $11.6 \pm 0.62 \%$ of the total cell count (Fig. 1).

Taurine ameliorates the decreased activities of antioxidant enzymes and increased lipid peroxidation induced by iron overload. SOD, catalase and GSH-Px are the important antioxidant enzymes present in the body that provide a defensive mechanism against free radical-mediated oxidative damage. Excess iron levels affect the activities of these enzymes as a result of the overproduction of ROS. The antioxidant activity of SOD is mediated by a dismutation reaction, in which SOD scavenges highly reactive superoxide radicals and converts them to oxygen molecules and less reactive $\mathrm{H}_{2} \mathrm{O}_{2}$ molecules (27). Catalase then further metabolizes the $\mathrm{H}_{2} \mathrm{O}_{2}$ into water and $\mathrm{O}_{2}$. The intracellular redox status is also maintained by the activity of GSH-Px in the presence of glutathione. GSH-Px aids in the decomposition of $\mathrm{H}_{2} \mathrm{O}_{2}$ and other organic hydroperoxides into non-toxic products (28). It has been observed in a number of previous studies that liver injury is associated with a reduction in the activities of these antioxidant enzymes $(29,30)$. Therefore, in the present study, the effect of iron overload on the activities of different antioxidant enzymes was investigated. It was found that there was 
Table I. Effect of taurine on the serum and hepatic iron concentration, liver-to-body weight ratio, serum levels of ALT and AST and hepatic taurine levels in iron-injected mice.

\begin{tabular}{|c|c|c|c|c|}
\hline Parameter & Placebo + vehicle & Placebo + taurine & Iron + vehicle & Iron + taurine \\
\hline Serum iron concentration $(\mu \mathrm{mol} / \mathrm{l})$ & $34.64 \pm 1.32$ & $32.80 \pm 1.41^{\mathrm{a}}$ & $420.36 \pm 15.42^{b}$ & $401.2 \pm 13.82^{\mathrm{b}, \mathrm{c}}$ \\
\hline Hepatic iron concentration (mg/g dry weight) & $0.068 \pm 0.003$ & $0.062 \pm 0.003^{\mathrm{a}}$ & $1.042 \pm 0.026^{\mathrm{b}}$ & $1.021 \pm 0.028^{\mathrm{b}, \mathrm{c}}$ \\
\hline Liver-to-body ratio (mg/g) & $48.2 \pm 1.9$ & $46.8 \pm 2.1^{\mathrm{a}}$ & $92.4 \pm 4.1^{\mathrm{b}}$ & $61.5 \pm 2.5^{\mathrm{b}, \mathrm{d}}$ \\
\hline $\operatorname{ALT}(\mathrm{U} / \mathrm{l})$ & $50.81 \pm 1.52$ & $48.83 \pm 1.65^{\mathrm{a}}$ & $228.31 \pm 9.42^{\mathrm{b}}$ & $125.06 \pm 5.33^{\mathrm{b}, \mathrm{d}}$ \\
\hline $\operatorname{AST}(\mathrm{U} / \mathrm{l})$ & $106.20 \pm 3.58$ & $113.42 \pm 3.91^{\mathrm{a}}$ & $395.13 \pm 14.22^{\mathrm{b}}$ & $216.42 \pm 8.23^{\mathrm{b,d}}$ \\
\hline Taurine level $(\mu \mathrm{mol} / \mathrm{g})$ & $30.03 \pm 1.51$ & $49.16 \pm 2.63^{\mathrm{e}}$ & $28.52 \pm 1.62^{\mathrm{a}}$ & $47.38 \pm 2.85^{\mathrm{d}, \mathrm{f}}$ \\
\hline
\end{tabular}

Data are expressed as the mean \pm the standard error of the mean $(\mathrm{n}=12)$. ${ }^{\mathrm{a}} \mathrm{P}>0.05$ vs. the placebo + vehicle group; ${ }^{\mathrm{b}} \mathrm{P}<0.01 \mathrm{vs}$. the placebo + vehicle and placebo + taurine groups; ${ }^{\mathrm{C}} \mathrm{P}>0.05$ vs. the iron + vehicle group; ${ }^{d} \mathrm{P}<0.01$ vs. the iron + vehicle group; ${ }^{\mathrm{e}} \mathrm{P}<0.01$ vs. the placebo + vehicle group and ${ }^{\mathrm{f}} \mathrm{P}>0.05$ vs. the placebo + taurine group. ALT, alanine transaminase; AST, aspartate transaminase.
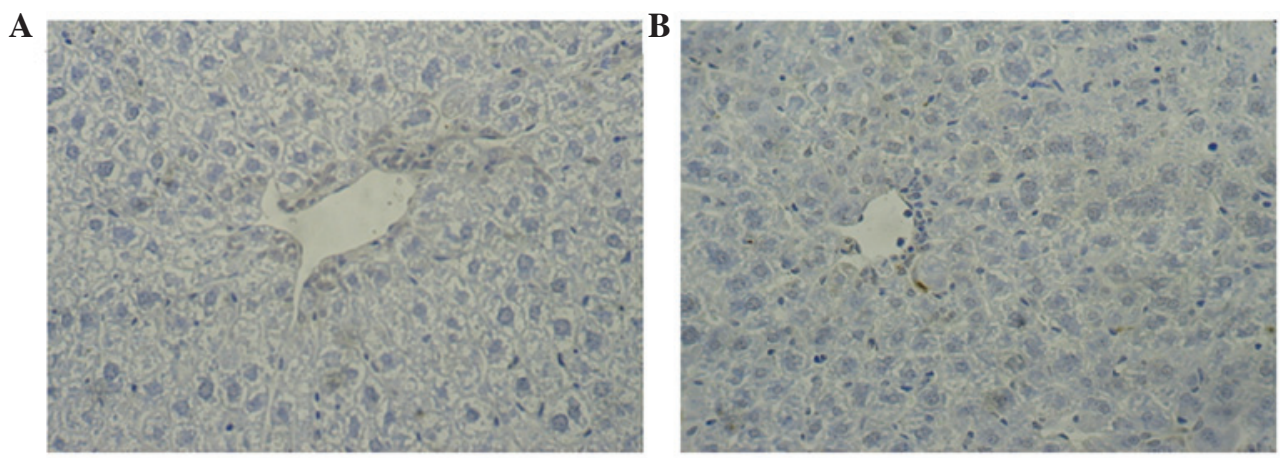

C

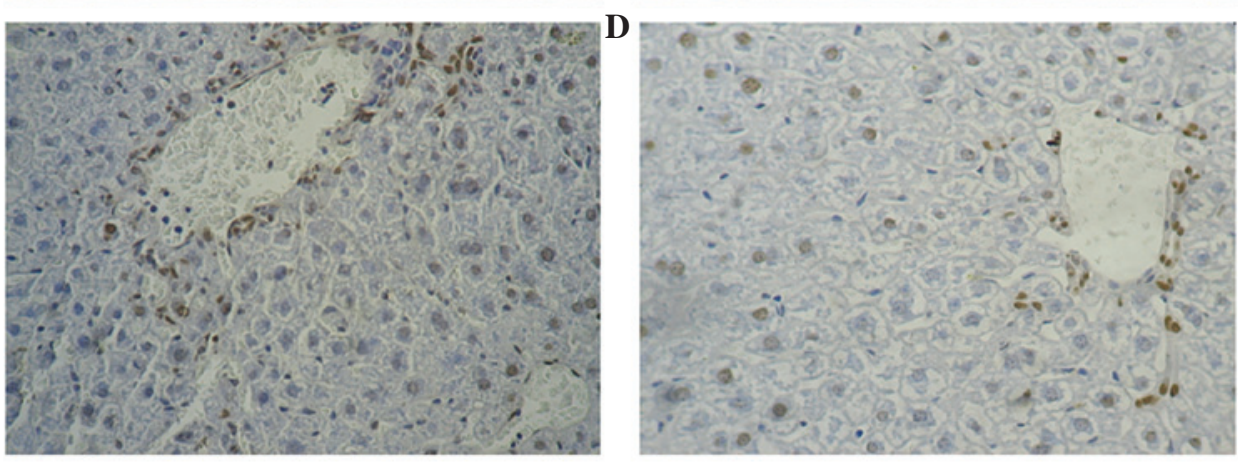

$\mathbf{E}$

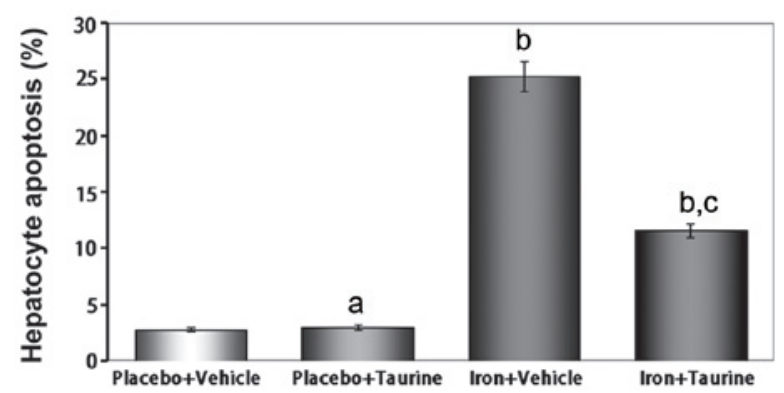

Figure 1. Effect of taurine on hepatocyte apoptosis in iron-overloaded mice. TUNEL-positive cells were apoptotic. (A-D) Liver sections from the different treatment groups: (A) placebo + vehicle, (B) placebo + taurine, (C) iron + vehicle and (D) iron + taurine (magnification, x400). (E) Quantitative analysis of hepatocyte apoptosis expressed as the percentage of TUNEL-positive nuclei among the hepatocytes. Data are presented as the mean \pm standard error of the mean $(\mathrm{n}=12)$. ${ }^{\mathrm{P}} \mathrm{P}>0.05 \mathrm{vs}$. the placebo + vehicle group; ${ }^{\mathrm{b}} \mathrm{P}<0.01$ vs. the placebo + vehicle and placebo + taurine groups and ${ }^{\mathrm{c}} \mathrm{P}<0.01 \mathrm{vs}$. the iron + vehicle group. TUNEL, terminal deoxynucleotidyl transferase-mediated dUTP nick end labeling.

a significant decrease in the activity of the enzymes analyzed; however, treatment with taurine increased the activities of SOD, catalase and GSH-Px in the hepatic tissue of the mice (Fig. 2).
Lipid peroxidation is believed to be one of the most important parameters of oxidative stress and is measured by estimating the MDA concentration, a lipid peroxidation end 
A

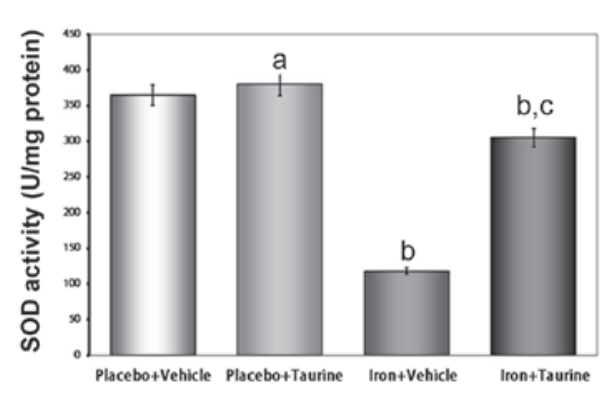

C

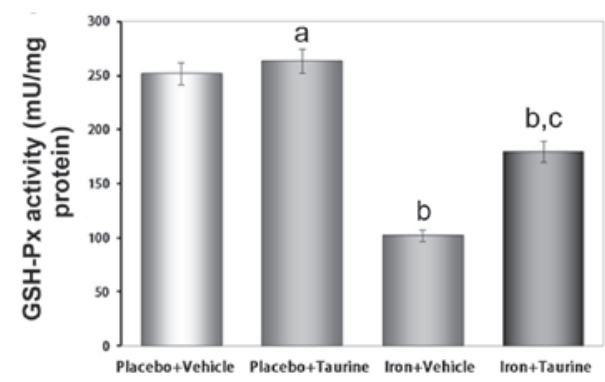

B

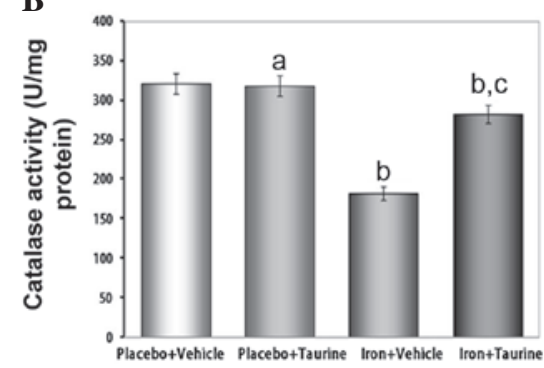

D

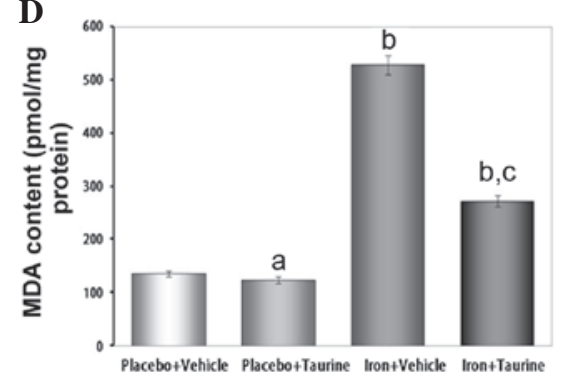

Figure 2. Effect of taurine on the activities of the antioxidant enzymes and lipid peroxidation in mice injected with iron over a 13-week period. (A-C) Activity of (A) SOD, (B) catalase, (C) GSH-Px and (D) MDA content. Data are presented as the mean \pm standard error of the mean ( $\mathrm{n}=12$ ). ${ }^{\mathrm{a}} \mathrm{P}>0.05 \mathrm{vs}$. the placebo + vehicle group; ${ }^{\mathrm{b}} \mathrm{P}<0.01$ vs. the placebo + vehicle and placebo + taurine groups and ${ }^{\mathrm{c}} \mathrm{P}<0.01$ vs. the iron + vehicle group. $\mathrm{SOD}$, superoxide dismutase; GSH-Px, glutathione-peroxidase; MDA, malondialdehyde.
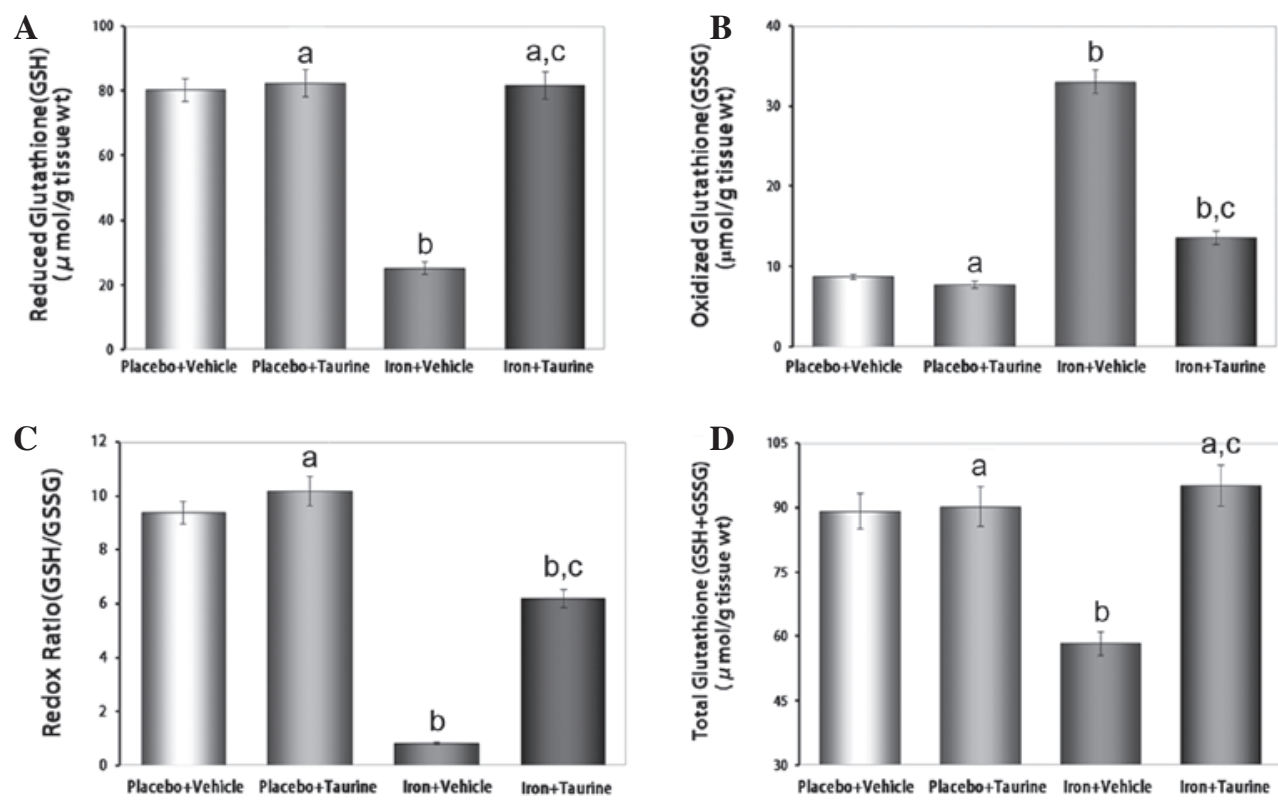

Figure 3. Effect of taurine on hepatic (A) GSH and (B) GSSG levels, (C) the redox ratio (GSH/GSSG) and (D) GSH+GSSG levels in mice injected with iron over a 13 -week period. Data are presented as the mean \pm standard error of the mean $(\mathrm{n}=12)$. ${ }^{\mathrm{a}} \mathrm{P}>0.05 \mathrm{vs}$. the placebo + vehicle group; ${ }^{\text {b }} \mathrm{P}<0.01 \mathrm{vs}$. the placebo + vehicle and placebo + taurine groups and ${ }^{\mathrm{C}} \mathrm{P}<0.01$ vs. the iron + vehicle group. GSH, glutathione; GSSG, glutathione disulfide.

product. In the present study, increased levels of MDA were observed in the iron-overloaded livers of experimental animals; however, taurine treatment was effective in preventing the extreme iron-induced alterations. These results suggest that the protective effects of taurine in the liver are correlated with the reduction in iron-induced oxidative stress.

Taurine protects GSH levels in iron-overloaded mice. Reduced glutathione, a ubiquitous tripeptide thiol, is an important intracellular metabolite that acts as an antioxidant and provides a secondary line of defense against intracellular free radicals and peroxides generated by oxidative stress (31). The reduced state of the cell is maintained by a high GSH/GSSG ratio. Owing to the potent antioxidant properties of taurine, it was hypothesized that taurine had the potential to preserve this high GSH/GSSG ratio in conditions of increased oxidative stress (32). It was observed that taurine supplementation in iron-overloaded mice almost entirely prevented the decrease in GSH $(\mathrm{P}<0.01)$ and increase in GSSG $(\mathrm{P}<0.01)$ levels, thereby partially protecting the redox ratio and normalizing the GSH+GSSG levels (Fig. 3). 


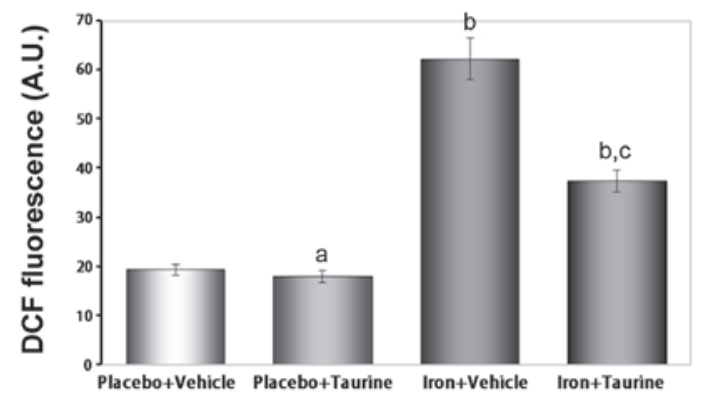

Figure 4. Effects of taurine on hepatic intercellular reactive oxygen species generation in mice injected with iron over a 13 -week period. Data are presented as the mean \pm standard error of the mean $(\mathrm{n}=12)$. ${ }^{\mathrm{a}} \mathrm{P}>0.05$ vs. the placebo + vehicle group; ${ }^{b} \mathrm{P}<0.01$ vs. the placebo + vehicle and placebo + taurine groups and ${ }^{\mathrm{c}} \mathrm{P}<0.01$ vs. the iron + vehicle group. $\mathrm{DCF}$, dichloro-dihydro-fluorescein.

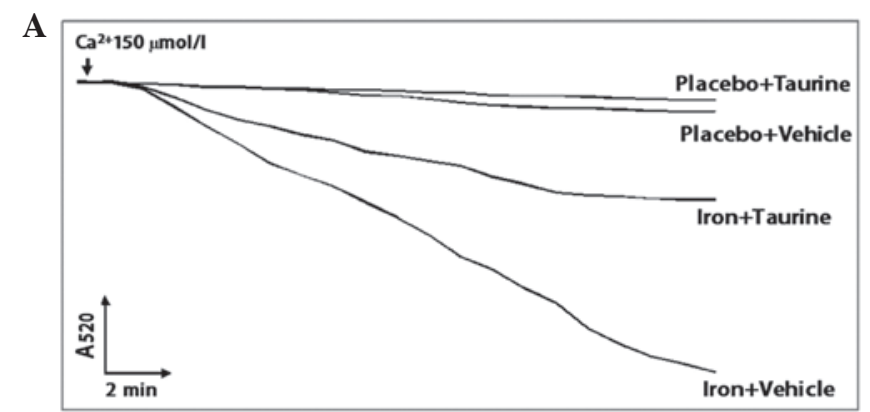

$\mathbf{B}$

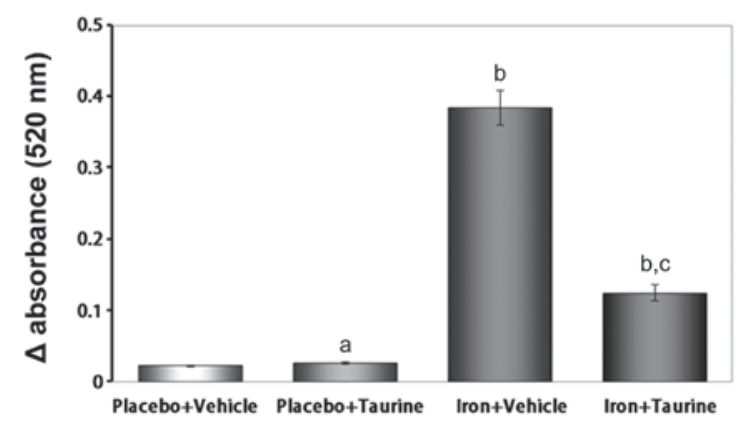

Figure 5. Effect of taurine on hepatocyte mitochondrial swelling in mice injected with iron over a 13 -week period. Data are presented as the mean \pm standard error of the mean $(n=12)$. (A) $\mathrm{Ca}^{2+}$-induced mitochondrial swelling in iron-overloaded mice, with or without taurine supplementation. (B) Quantitative analysis of mitochondrial swelling. ${ }^{\mathrm{P}} \mathrm{P}>0.05$ vs. the placebo + vehicle group; ${ }^{\mathrm{P}} \mathrm{P}<0.01$ vs. the placebo + vehicle and placebo + taurine groups and ${ }^{\mathrm{C}} \mathrm{P}<0.01$ vs. the iron + vehicle group.

Taurine inhibits ROS generation in iron-overloaded mice. It is well established that iron induces toxicity in the liver, as iron is primarily stored in the liver and produces ROS (33). Therefore, the intracellular ROS levels were measured using a DCFH-DA assay. The ROS levels were observed to be significantly increased in the iron-treated animals compared with those in the untreated animals $(\mathrm{P}<0.01)$. This indicates that supplementation with taurine significantly prevents ROS formation induced by iron overload (Fig. 4).

Taurine inhibits mitochondrial swelling in iron-overloaded mice. Iron-induced damage to the inner mitochondrial membrane may be assessed using classic swelling techniques, which monitor the net influx of the osmotic support associated

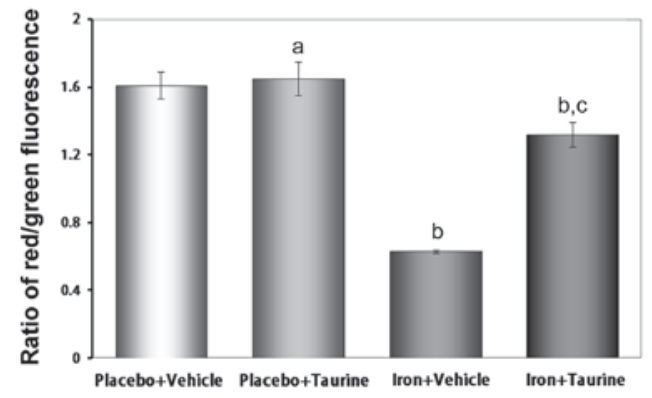

Figure 6. Effect of taurine on the mitochondrial membrane potential in mice injected with iron over a 13 -week period. Data are presented as the mean \pm standard error of the mean $(n=12)$. ${ }^{a} \mathrm{P}>0.05$ vs. the placebo + vehicle group; ${ }^{b} \mathrm{P}<0.01$ vs. placebo + vehicle and placebo + taurine groups and ${ }^{\mathrm{c}} \mathrm{P}<0.01$ vs. the iron + vehicle group.

with a non-specific increase in membrane permeability. It was shown that iron induced mitochondrial swelling, indicated by the decrease in the absorbance of the mitochondrial suspension at $520 \mathrm{~nm}$. However, taurine inhibited this swelling process (P<0.01) (Fig. 5).

Taurine attenuates the loss of the $\Delta \psi$ in iron-overloaded mice. The loss of the $\Delta \psi$ is an early indicator of apoptosis. The unique fluorescent cationic dye JC-1 was used to measure the $\Delta \psi$ in the mitochondria of hepatocytes. In healthy cells, where the $\Delta \psi$ is normal, JC- 1 accumulates as aggregates in the mitochondrial matrix, where it emits red fluorescence. In apoptotic and dead cells, where the $\Delta \psi$ is lost, JC-1 exists in a monomeric form and emits green fluorescence. Therefore, the red to green fluorescence intensity ratio may be used to evaluate the $\Delta \psi$. As shown in Fig. 6, the iron plus vehicle group exhibited a decrease in the ratio of red to green fluorescence intensity $(\mathrm{P}<0.01)$, indicating a loss of the $\Delta \psi$. However, treatment with taurine significantly attenuated the loss of the $\Delta \psi(\mathrm{P}<0.01)$.

\section{Discussion}

Previous studies in humans and animals have shown that iron overload is a risk factor for liver damage (34). Although oxidative stress is considered to be a major cause of hepatotoxicity induced by iron overload, the underlying mechanism remains unknown. In the present study, mice that were injected with iron exhibited marked symptoms of iron toxicity, including an elevated liver-to-body weight ratio, increased levels of AST and ALT in the plasma and serum and intracellular iron deposition in the liver, concurrent with increased apoptosis.

Free radical production and oxidative stress play key roles in the initiation and progression of iron overload hepatotoxicity (35). In the livers of iron-overloaded mice, marked increases in ROS and MDA (an end product of lipid peroxidation) levels were observed, as well as a depletion of GSH and GSH+GSSG levels. These findings were indicative of increased oxidative damage. Free radical-induced lipid peroxidation leads to cellular dysfunction. Clinically, the AST and ALT levels in the plasma represent biomarkers for liver function (36). In the present study, the serum AST and ALT levels in the mice were significantly elevated following 
iron overload, suggesting an iron overload-related injury to the liver.

Taurine is a conditionally essential amino acid that contains a sulfonic acid group and has several physiological roles $(37,38)$. Increasing evidence has shown that taurine exerts strong protective effects due to its antioxidant characteristics $(17,39,40)$. In the present study taurine levels in the liver were increased to investigate whether this prevented iron overload-induced hepatic damage, despite the fact that taurine has no effect on iron deposition. This rationale was based on the finding that there is an increased demand for taurine in instances of oxidative stress. The results showed that taurine supplementation was associated with the protection of liver function in iron-overloaded mice. The protective effects of taurine occurred concurrently with a reduction in apoptosis, suggesting that taurine was able to decrease the toxic effects of iron. The protective effects of taurine have been previously associated with its antioxidant properties. Furthermore, taurine has been shown to reduce iron-mediated lipid peroxidation and increase the activities of antioxidant enzymes (41).

Consistent with the antioxidant properties of taurine observed in the present study, a marked reduction in MDA formation was observed in the iron-overloaded mice. The potent antioxidant properties of taurine are additionally associated with increased antioxidant enzyme activity. SOD, catalase and GSH-Px are the key cellular antioxidant enzymes that defend against oxidative stress. Evidence shows that the activities of these antioxidant enzymes are decreased when cells and tissues are subjected to oxidative stress $(42,43)$. The antioxidant effects attributed to taurine may be associated with its sulfur moiety, and the modulation of GSH and GSH levels by taurine is critical in the cellular defense against oxidative stress $(44,45)$. During oxidative stress, increased cellular demand leads to the depletion of GSH and the accumulation of GSSG. As a result, a shift in the redox state occurs and the cell function becomes impaired. In the present study, taurine supplementation completely prevented the iron-induced depletion in the GSH levels while protecting the redox ratio. These findings elucidate a possible mechanism underlying the actions of taurine and may explain its pleiotropic and beneficial effects following an increase in oxidative stress $(42,43)$.

Hepatocyte damage mediated by oxidative stress is associated with the disruption of the $\Delta \psi(46)$. Mitochondria are important organelles that have a vital role in apoptosis (47). The mitochondrial permeability transition pore (mPTP) is a multiprotein complex that can form large, nonselective pores in the inner mitochondrial membrane (48). ROS are one of the most important factors stimulating the opening of the mPTPs. If the mPTPs are constantly open, mitochondrial swelling can occur, resulting in the rupture of the outer mitochondrial membrane and culminating in apoptosis. The present study demonstrated that overproduction of ROS in iron-overloaded mice increased the mitochondrial membrane permeability, induced the opening of the mPTPs, and resulted in mitochondrial swelling and depolarization (49). Therefore, the collapse in $\Delta \psi$ may be a consequence of the oxidative stress in hepatocytes. Taurine treatment led to a reduction in the liver ROS levels due to its strong ROS scavenging and antioxidant properties, as previously mentioned. Reduced ROS levels prevented the MPTPs from opening and the subsequent mitochondrial swelling, which blocked $\Delta \psi$ depolarization.

In conclusion, the murine model of an iron-overloaded liver used in the present study demonstrated that taurine supplementation has beneficial effects on liver function and apoptosis, with marked reductions in oxidative stress induced by iron overload. Due to the benefits provided and the absence of toxicity with taurine supplementation, increased dietary intake of taurine represents an important nutritional modification that could be a useful therapeutic alternative to reduce the hepatic toxicity induced by iron overload in such diseases as hemosiderosis.

\section{Acknowledgements}

This study was supported by grants from the Natural Scientific Foundation of China (nos. 30760075, 30860271, 81100104 and 81160309).

\section{References}

1. Santos PC, Krieger JE and Pereira AC: Molecular diagnostic and pathogenesis of hereditary hemochromatosis. Int J Mol Sci 13: 1497-1511, 2012.

2. Murphy CJ and Oudit GY: Iron-overload cardiomyopathy: pathophysiology, diagnosis, and treatment. J Card Fail 16: 888-900, 2010.

3. Moretti D, van Doorn GM, Swinkels DW and Melse-Boonstra A: Relevance of dietary iron intake and bioavailability in the management of HFE hemochromatosis: a systematic review. Am J Clin Nutr 98: 468-479, 2013.

4. Alústiza Echeverría JM, Castiella A and Emparanza JI: Quantification of iron concentration in the liver by MRI. Insights Imaging 3: 173-180, 2012.

5. Lee M and Kowdley KV: Alcohol's effect on other chronic liver diseases. Clin Liver Dis 16: 827-837, 2012.

6. Hayashi H, Piperno A, Tomosugi N, et al: Patients with chronic hepatitis $\mathrm{C}$ may be more sensitive to iron hepatotoxicity than patients with HFE-hemochromatosis. Intern Med 49: 2371-2377, 2010.

7. Abdalla MY, Fawzi M, Al-Maloul SR, El-Banna N, Tayyem RF and Ahmad IM: Increased oxidative stress and iron overload in Jordanian $\beta$-thalassemic children. Hemoglobin 35: 67-79, 2011

8. Li X, Li H, Lu N, Feng Y, Huang Y and Gao Z: Iron increases liver injury through oxidative/nitrative stress in diabetic rats: Involvement of nitrotyrosination of glucokinase. Biochimie 94: 2620-2627, 2012.

9. Serviddio G, Bellanti F, Sastre J, Vendemiale G and Altomare E: Targeting mitochondria: a new promising approach for the treatment of liver diseases. Curr Med Chem 17: 2325-2337, 2010.

10. Liu D, He H, Yin D, Que A, Tang L, Liao Z, Huang Q and He M: Mechanism of chronic dietary iron overload-induced liver damage in mice. Mol Med Rep 7: 1173-1179, 2013.

11. Batista TM, Ribeiro RA, da Silva PM, Camargo RL, Lollo PC, Boschero AC and Carneiro EM: Taurine supplementation improves liver glucose control in normal protein and malnourished mice fed a high-fat diet. Mol Nutr Food Res 57: 423-434, 2013.

12. Heidari R, Babaei H and Eghbal MA: Cytoprotective effects of taurine against toxicity induced by isoniazid and hydrazine in isolated rat hepatocytes. Arh Hig Rada Toksikol 64: 15-24, 2013.

13. Hartman JC, Brouwer K, Mandagere A, Melvin L and Gorczynski R: Evaluation of the endothelin receptor antagonists ambrisentan, darusentan, bosentan, and sitaxsentan as substrates and inhibitors of hepatobiliary transporters in sandwich-cultured human hepatocytes. Can J Physiol Pharmacol 88: 682-691, 2010.

14. Liao Y, Lu X, Lu C, Li G, Jin Y and Tang H: Selection of agents for prevention of cisplatin-induced hepatotoxicity. Pharmacol Res 57: 125-131, 2008.

15. Tabassum H, Rehman H, Banerjee BD, Raisuddin S and Parvez S: Attenuation of tamoxifen-induced hepatotoxicity by taurine in mice. Clin Chim Acta 370: 129-136, 2006.

16. Wang GG, Li W, Lu XH, Zhao X and Xu L: Taurine attenuates oxidative stress and alleviates cardiac failure in type I diabetic rats. Croat Med J 54: 171-179, 2013. 
17. Higuchi M, Celino FT, Shimizu-Yamaguchi S, Miura C and Miura T: Taurine plays an important role in the protection of spermatogonia from oxidative stress. Amino Acids 43: 2359-2369, 2012.

18. Oudit GY, Sun H, Trivieri MG, Koch SE, Dawood F, Ackerley C, Yazdanpanah M, Wilson GJ, Schwartz A, Liu PP and Backx PH: L-type $\mathrm{Ca}^{2+}$ channels provide a major pathway for iron entry into cardiomyocytes in iron-overload cardiomyopathy. Nat Med 9: 1187-1194, 2003

19. Galleano M and Puntarulo S: Hepatic chemiluminescence and lipid peroxidation in mild iron overload. Toxicology 76: 27-38, 1992.

20. Keith ME, Ball A, Jeejeebhoy KN, Kurian R, Butany J, Dawood F, Wen WH, Madapallimattam A and Sole MJ Conditioned nutritional deficiencies in the cardiomyopathic hamster heart. Can J Cardiol 17: 449-458, 2001.

21. Okhawa $\mathrm{H}$, Ohishi $\mathrm{N}$ and Yagi K: Assay for lipid peroxides in animal tissues by thiobarbituric acid reaction. Anal Biochem 95: 351-358, 1979

22. Beauchamp C and Fridovich I: Superoxide dismutase: improved assays and an assay applicable to acrylamide gels. Anal Biochem 44: 276-287, 1971

23. Hill MF and Singal PK: Right and left myocardial antioxidant responses during heart failure subsequent to myocardial infarction. Circulation 96: 2414-2420, 1997.

24. Anderson ME: Determination of glutathione and glutathione disulfide in biological samples. Methods Enzymol 113: 548-555, 1985.

25. Kalantari-Dehaghi M, Chen Y, Deng W, Chernyavsky A Marchenko S, Wang PH and Grando SA: Mechanisms of mitochondrial damage in keratinocytes by Pemphigus vulgaris antibodies. J Biol Chem 288: 16916-16925, 2013

26. Beavis AD, Brannan RD and Garlid KD: Swelling and contraction of the mitochondrial matrix. I. A structural interpretation of the relationship between light scattering and matrix volume. J Biol Chem 260: 13424-13433, 1985.

27. Misra HP and Fridovich I: The role of superoxide anion in the autoxidation of epinephrine and a simple assay for superoxide dismutase. J Biol Chem 247: 3170-3175, 1972.

28. Ketterer B: Detoxication reactions of glutathione and glutathione transferases. Xenobiotica 16: 957-973, 1986.

29. Liu CM, Zheng GH, Ming QL, Sun JM and Cheng C: Protective effect of quercetin on lead-induced oxidative stress and endoplasmic reticulum stress in rat liver via the IRE1/JNK and PI3K/Akt pathway. Free Radic Res 47: 192-201, 2013.

30. Banu S, Bhaskar B and Balasekar P: Hepatoprotective and antioxidant activity of Leucas aspera against D-galactosamine induced liver damage in rats. Pharm Biol 50: 1592-1595, 2012.

31. Pompella A, Visvikis A, Paolicchi A, De Tata V and Casini AF: The changing faces of glutathione, a cellular protagonist. Biochem Pharmacol 66: 1499-1503, 2003.

32. Schaffer SW, Azuma J and Mozaffari M: Role of antioxidant activity of taurine in diabetes. Can J Physiol Pharmacol 87 : 91-99, 2009
33. Pardo Andreu GL, Inada NM, Vercesi AE and Curti C: Uncoupling and oxidative stress in liver mitochondria isolated from rats with acute iron overload.Arch Toxicol 83: 47-53, 2009.

34. Tirnitz-Parker JE, Glanfield A, Olynyk JK and Ramm GA: Iron and hepatic carcinogenesis. Crit Rev Oncog 18: 391-407, 2013.

35. Sorrentino P, Terracciano L, D'Angelo S, et al: Oxidative stress and steatosis are cofactors of liver injury in primary biliary cirrhosis. J Gastroenterol 45: 1053-1062, 2010.

36. Lalisang TJ: Serum bile acid: an alternative liver function marker in the obstructive jaundice patient. Acta Med Indones 44 233-238, 2012.

37. Manna P, Sinha M and Sil PC: Taurine plays a beneficial role against cadmium-induced oxidative renal dysfunction. Amino Acids 36: 417-428, 2009.

38. Sinha M, Manna P and Sil PC: Taurine, a conditionally essential amino acid, ameliorates arsenic-induced cytotoxicity in murine hepatocytes. Toxicol In Vitro 21: 1419-1428, 2007.

39. Aruoma OI, Halliwell B, Hoey BM and Butler J: The antioxidant action of taurine, hypotaurine and their metabolic precursors. Biochem J 256: 251-255, 1988.

40. Budhram R, Pandya KG and Lau-Cam CA: Protection by taurine and thiotaurine against biochemical and cellular alterations induced by diabetes in a rat model. Adv Exp Med Biol 775: 321-343, 2013

41. Kang IS and Kim C: Taurine chloramine administered in vivo increases NRF2-regulated antioxidant enzyme expression in murine peritoneal macrophages. Adv Exp Med Biol 775: 259-267, 2013.

42. Ghyasi R, Sepehri G, Mohammadi M, Badalzadeh R and Ghyasi A: Effect of mebudipine on oxidative stress and lipid peroxidation in myocardial ischemic-reperfusion injury in male rat. J Res Med Sci 17:1150-1155, 2012.

43. Ramesh B, Karuna R, Sreenivasa RS, Haritha K, Sai MD, Sasi BR and Saralakumari D: Effect of Commiphora mukul gum resin on hepatic marker enzymes, lipid peroxidation and antioxidants status in pancreas and heart of streptozotocin induced diabetic rats. Asian Pac J Trop Biomed 2: 895-900, 2012.

44. Huxtable RJ: Physiological actions of taurine. Physiol Rev 72: 101-163, 1992.

45. Woo HA, Chae HZ, Hwang SC, Yang KS, Kang SW, Kim K and Rhee SG: Reversing the inactivation of peroxiredoxins caused by cysteine sulfinic acid formation. Science 300: 653-656, 2003.

46. Sharma V, Anderson D and Dhawan A: Zinc oxide nanoparticles induce oxidative DNA damage and ROS-triggered mitochondria mediated apoptosis in human liver cells (HepG2). Apoptosis 17: 852-870, 2012

47. Zhao CY, Liu XY and Shen C: Molecular mechanisms of mitochondrial damage in the development of liver failure. Zhonghua Gan Zang Bing Za Zhi 19: 955-957, 2011 (In Chinese).

48. Gottlieb RA and Gustafsson AB: Mitochondrial turnover in the heart. Biochim Biophys Acta 1813: 1295-1301, 2011.

49. Bae YS, Oh H, Rhee SG and Yoo YD: Regulation of reactive oxygen species generation in cell signaling. Mol Cells 32: 491-509, 2011. 